Int. J. Dev. Biol. 59: 241-246 (2015)

doi: $10.1387 /$ ijdb.140263ho

\title{
Identification of distal enhancers for Six2 expression in pronephros
}

\author{
NANOKA SUZUKI ${ }^{1}$, KODAI HIRANO ${ }^{1}$, HAJIME OGINO ${ }^{2,3}$ and HARUKI OCHI*,1 \\ ${ }^{1}$ Faculty of Medicine, Yamagata University, Yamagata-shi, Yamagata, \\ ${ }^{2}$ Department of Animal Bioscience, Nagahama Institute of Bio-Science and Technology, Nagahama-shi, Shiga and \\ 3JST, CREST, 5, Sanbancho, Chiyoda-ku, Tokyo, Japan
}

\begin{abstract}
The embryonic nephric mesenchyme contains pluripotent progenitor cells. Six2, a homeodomain transcription factor, is expressed in a subset of the nephric mesenchyme, and it functions to maintain a progenitor state by suppressing nephrogenesis. Despite the functional significance of Six2 in nephric development, its regulatory mechanisms remain unclear. To identify the cis-regulatory elements for Six2, we focused on the evolutionarily conserved sequences known as conserved noncoding sequences (CNSs) associated with the Six2 locus. Transgenic experiments using Xenopus laevis embryos revealed that three of the eight CNSs located within a 317-kb segment of the Six 2 genomic locus were nephric enhancers. Motif analysis of transcription factors combined with phylogenetic footprinting revealed the enrichment of putative T-cell factor (Tcf)-, Hox-, and SWI/SNF complex helicase-like transcription factor (HItf)- and AT-rich interactive domain 3A (Arid3a)-binding motif sequences in these enhancers.
\end{abstract}

KEY WORDS: Six2, conserved noncoding sequence, nephric enhancer, Xenopus laevis, transgenic system

\section{Introduction}

Six2 is a vertebrate homolog of the Drosophila homeobox gene sine oculis and is expressed in developing neural tissues, such as the eyes, otic placodes, and cranial ganglia, and in non-neural tissues, such as the head mesenchyme, somite, muscle anlagen, and mesenchyme associated with the nephron in Xenopusembryos (Ghanbari et al., 2001). Targeted disruption of Six2 in mouse embryos results in ectopic premature epithelialization of the nephric mesenchyme (Self et al., 2006). Moreover, Six2 was recently found to control both the self-renewal of nephron progenitors and the commitment to nephric lineages with Wnt-signaling components, $\beta$-catenin and lymphoid enhancer factor (Lef)/T-cell factor (Tcf) in mouse embryos (Park et al., 2012). Although these studies have illustrated the essential roles of Six 2 in the early steps of nephrogenesis, its regulatory mechanisms remain unclear.

Cis-regulatory analysis of a key regulatory gene is a straightforward approach for understanding the mechanisms of tissue induction and development. However, because some enhancers are located distantly from their target genes, identification via traditional genome walking was a slow and painstaking process.
This situation has recently been changed by the development of comparative genomics approaches, which discovered a number of evolutionarily conserved noncoding sequences (CNSs) associated with developmental genes (Woolfe et al., 2005). Transgenic analyses using mice, zebrafish, and frogs have revealed that some CNSs are actually important enhancers and/or silencers for gene expression (Woolfe et al., 2005)(Ochi et al., 2012).

In this study, we identified eight CNSs that are conserved in tetrapods and are located within a 317-kb segment of the Xnopus tropicalis Six2 genomic locus. A transgenic reporter assay in Xenopus embryos revealed that three of these CNSs function as nephric enhancers. Phylogenetic footprinting analysis of these enhancers followed by motif searches identified putative Tcf-, Hox, helicase-like transcription factor (HItf)-, and AT-rich interactive domain $3 \mathrm{~A}$ (Arid3a)-binding sequences as the enriched motifs. These results suggest that the combination of input transcription factors, Tcf, Hox, and SWI/SNF complex on the distal enhancers drive Six2 expression in pronephros.

Abbreviations used in this paper: CNS, conserved noncoding sequence; GFP, green fluorescent protein.

\footnotetext{
*Address correspondence to: Haruki Ochi. Faculty of Medicine, Yamagata University, 2-2-2 lida-Nishi, Yamagata-shi, Yamagata 990-9585 Japan. Tel: +81-23-628-5925. Fax: +81-23-628-5925. E-mail: harukiochi@ med.id.yamagata-u.ac.jp
}

Accepted: 23 April 2015 


\section{Results and Discussion}

\section{Conserved noncoding sequences in the Six2 locus}

The homeodomain transcription factor Six2 expressed within the kidney mesenchyme suppresses nephrogenesis to maintain progenitor renewal (Ghanbari et al., 2001)(Self et al., 2006). An enhancer assay using cultured kidney cells revealed that the Hox-Eya-Pax complex regulates Six2 expression through a 450-bp genomic region upstream of the Six2 translational start site (Gong et al., 2007). A transgenic enhancer assay using mice also illustrated that a 1-kb segment upstream of Six2 containing the Hox-Eya-Pax complex-binding site drove reporter expression in kidney cells (Yallowitz et al., 2009). Although the cis-regulatory elements controlling developmental gene expression are often associated within the proximal region of the gene, some cisregulatory elements are known to have the ability to function over distances of $100 \mathrm{~kb}$ or longer (Kleinjan et al., 2006). To date, no distal enhancers for Six2 were reported, since the identification of such distal enhancer using traditional genome walking were very costly and laborious. The evolutionarily CNSs represent landmarks for identifying such distal enhancers (Woolfe et al., 2005)(Ochi et al., 2012). To explore the cis-regulatory candidates for the distal enhancers of Six2, we initially compared the genomic sequence of a 364-kb segment encompassing human SIX2 with the orthologous regions in the mouse, opossum, and frog ( $X$. tropicalis) genomes using the MulitPipMaker alignment tool (Schwartz, 2000). This analysis identified eight CNSs (CNS1-CNS8) as candidate Six2 cis-regulatory elements (Fig. 1A).

\section{Identification of Six2 nephric enhancers using a Xenopus transgenic reporter assay}

The $X$. laevis transgenic system is known as an efficient transgenesis technique for a non-mosaic founder assay and as a useful

\section{A}

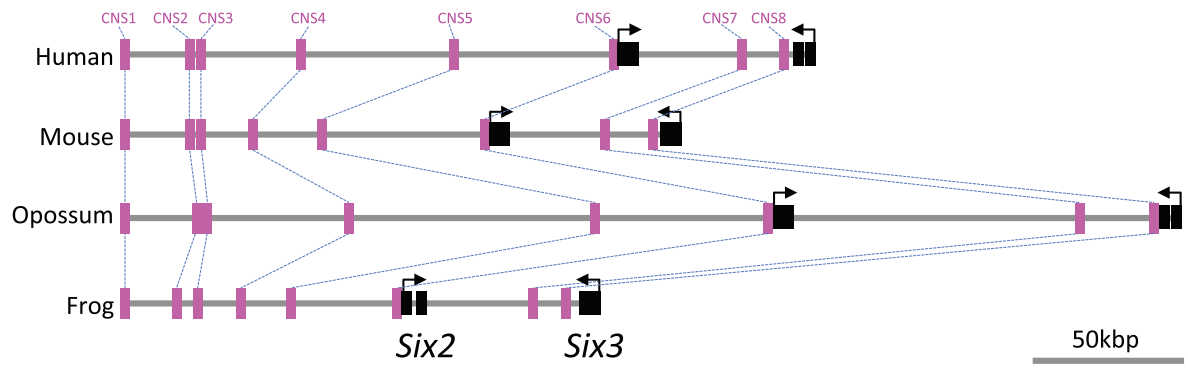

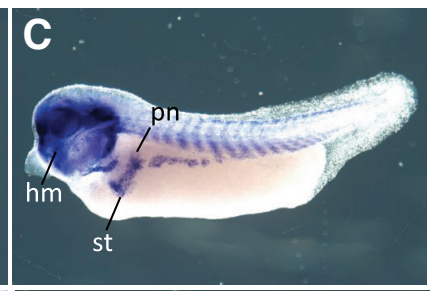

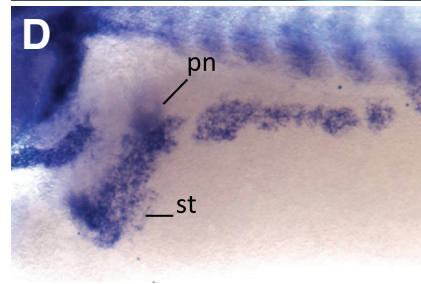

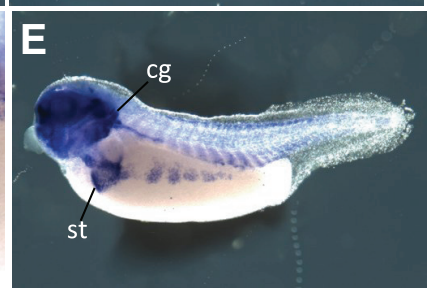

system to screen for cis-regulatory elements (Ogino and Ochi, 2009). Six2 from $X$. laevis, a tetraploid frog, is expressed in the region of the developing pronephric kidneys, resembling mouse Six2 expression (Ghanbari et al., 2001). A diploid frog $X$. tropicalis was recently developed as a model animal, and its genomic sequences are available. Hence, this genomic resource is suitable for enhancer screening using the $X$. laevis transgenic system. The expression of endogenous Six 2 in $X$. tropicalis embryo was first examined by in situ hybridization, and the results confirmed that its expression in pronephric kidneys closely resembled that of X.laevis (Fig. 1B-D)(Ghanbari et al., 2001). Each X. tropicalis Six2 CNS was cloned into a GFP reporter plasmid carrying a $\beta$ actin basal promoter. Each construct was then used to generate transgenic embryos, and their reporter expression was examined once the resulting embryos reached stage 35/36, at which point endogenous Six2 is expressed in the proximal tubule region (Fig. 1 C,D)(Ghanbari etal., 2001). The reporter constructs without a CNS displayed non-significant GFP expression (Table 1). Of the eight Six2 CNSs tested, three CNSs, CNS2, CNS3, and CNS5, drove strong and reproducible pronephric expression (Fig. $2 \mathrm{~B}, \mathrm{C}$, and $\mathrm{E}$; Table 1). CNS6 was immediately adjacent to Six2, for which the corresponding region previously identified as the nephric enhancer in mice displayed reporter expression in the pronephros, although the frequency of pronephric positive embryos was less than that of CNS2, CNS3, and CNS5 expression (Fig. 2F; Table 1)(Gong et al., 2007) (Yallowitz et al., 2009). CNS4 was also expressed in the pronephros, and its frequency of expression was less than those of CNS2, CNS3, and CNS5 (Fig. 2D; Table 1). CNS7, located downstream of Six2, drove GFP reporter expression in otic vesicles, in which endogenous Six2 is expressed (Fig. 2G; Table 1). CNS8, which was located near Six3, was active in the eye tissues (Fig. $2 \mathrm{H}$; Table 1). Transverse sections indicated that CNS2 and CNS3 drove strong GFP reporter expression both in the pronephric tubules and in the non-glomus mesenchyme, while CNS5 was mostly expressed in the pronephric tubules (Fig. 2. B",C",E"). Thus, we identified CNS2, CNS3, and CNS5 as nephricenhancers of Six2. These enhancers are located 82, 75, and 40 $\mathrm{kb}$ upstream of the Six2 transcription start site in the $X$. tropicalis genome (Fig. 1A). Shadow enhancers drive a pattern of transcription that resembles the drive pattern of previously identified primary enhancers (Barolo, 2012). According to this category, CNS6, immediately adjacent to Six2, is categorized as the primary enhancer, and CNS2, CNS3, and CNS5 are considered shadow enhancers (Barolo, 2012). Because shadow enhancers modulate pheno-

Fig. 1. Conservation of noncoding sequences across the Six2 locus in tetrapods. (A) Schematic overview of the Six2 locus. Magenta and black boxes indicate conserved noncoding sequences and exons, respectively. (B-D) In situ hybridization analysis illustrating the expression of Six2 in Xenopus tropicalis at the stages 26, 35/36, and 43 (B,C,E). Higher magnification image of stages 35/36 embryos (D) Pn, pronephros; st, stomach; $h m$, head mesenchyme; ov, otic vesicle; cg, cranial ganglia. 
TABLE 1

SCORING RESULTS FROM THE XENOPUS TRANSGENIC REPORTER ASSAY

\begin{tabular}{|c|c|c|c|c|c|c|c|c|c|c|}
\hline Construct & Analysed embryos (n) & Number of GFP positive (n) & Pronephrons & MHB & Neural tube & Eye & Otic Vesicle & Pharyngealarch & Somite & $\%$ of pronephric activity \\
\hline$\beta$-GFP & 82 & $\begin{array}{l}2 \\
1\end{array}$ & & & & + & & + & + & $0 \%$ \\
\hline Six2-CNS1- $\beta-G F P$ & 103 & $\begin{array}{l}5 \\
5 \\
3 \\
2 \\
1 \\
1 \\
1\end{array}$ & $\begin{array}{l}+ \\
+\end{array}$ & + & $\begin{array}{l}+ \\
+\end{array}$ & + & & + & $\begin{array}{l}+ \\
+ \\
+ \\
+ \\
+\end{array}$ & $3 \%$ \\
\hline Six2-CNS2- $\beta-G F P$ & 106 & $\begin{array}{c}10 \\
5 \\
3 \\
3 \\
2 \\
1 \\
1 \\
1\end{array}$ & $\begin{array}{l}+ \\
+ \\
+ \\
+\end{array}$ & $\begin{array}{l}+ \\
+ \\
+ \\
+\end{array}$ & + & & & $\begin{array}{l}+ \\
+ \\
+ \\
+ \\
+ \\
+ \\
+ \\
+\end{array}$ & + & $18 \%$ \\
\hline Six2-CNS3- $\beta$-GFP & 92 & $\begin{array}{c}18 \\
1 \\
1 \\
1\end{array}$ & + & + & + & + & & + & + & $19 \%$ \\
\hline Six2-CNS4- $\beta-G F P$ & 104 & $\begin{array}{l}4 \\
3 \\
3 \\
2 \\
2\end{array}$ & $\begin{array}{l}+ \\
+\end{array}$ & & & + & & $\begin{array}{l}+ \\
+\end{array}$ & $\begin{array}{l}+ \\
+ \\
+\end{array}$ & $6 \%$ \\
\hline Six2-CNS5- $\beta$-GFP & 142 & $\begin{array}{l}9 \\
6 \\
5 \\
3 \\
2 \\
1 \\
1 \\
1 \\
1 \\
1 \\
1\end{array}$ & $\begin{array}{l}+ \\
+ \\
+ \\
+ \\
+ \\
+ \\
+ \\
+\end{array}$ & & + & $\begin{array}{l}+ \\
+\end{array}$ & $\begin{array}{l}+ \\
+\end{array}$ & $\begin{array}{l}+ \\
+ \\
+\end{array}$ & $\begin{array}{l}+ \\
+ \\
+ \\
+ \\
+ \\
+ \\
+ \\
+\end{array}$ & $22 \%$ \\
\hline Six2-CNS6- $\beta$-GFP & 89 & $\begin{array}{l}4 \\
2 \\
1\end{array}$ & $\begin{array}{l}+ \\
+ \\
+\end{array}$ & & + & & & $\begin{array}{l}+ \\
+\end{array}$ & + & $8 \%$ \\
\hline Six2-CNS7- $\beta$-GFP & 74 & $\begin{array}{l}3 \\
2 \\
2 \\
1 \\
1 \\
1\end{array}$ & $\begin{array}{l}+ \\
+ \\
+\end{array}$ & $\begin{array}{l}+ \\
+\end{array}$ & & $\begin{array}{l}+ \\
+ \\
+ \\
+\end{array}$ & $\begin{array}{l}+ \\
+ \\
+\end{array}$ & $\begin{array}{l}+ \\
+ \\
+ \\
+ \\
+\end{array}$ & + & $5 \%$ \\
\hline Six2-CNS8- $\beta$-GFP & 133 & $\begin{array}{c}13 \\
9 \\
4 \\
3 \\
2 \\
1 \\
1 \\
\end{array}$ & $\begin{array}{l}+ \\
+\end{array}$ & & & $\begin{array}{l}+ \\
+ \\
+ \\
+ \\
+ \\
+ \\
+\end{array}$ & & $\begin{array}{l}+ \\
+ \\
+ \\
+\end{array}$ & $\begin{array}{l}+ \\
+ \\
+\end{array}$ & $6 \%$ \\
\hline
\end{tabular}

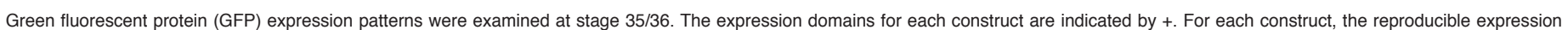

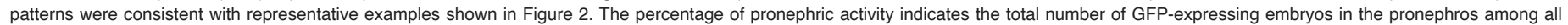
examined embryos. MHB, midbrain-hindbrain boundary

typic robustness, Six2 distal nephric enhancers may function as redundant enhancers (Barolo, 2012).

\section{Characterization of the candidate transcription inputs}

To dissect the transcriptional inputs for the Six2 nephric enhancers, the open-access database JASPAR ver. 5 was used to define potential transcription factor-binding sites (Mathelier et al., 2014). The candidate transcription factors were narrowed on the basis of their nephric expressions using the Expression Atlas (the baseline atlas)(Petryszak et al., 2014). Finally, candidate transcription factors were narrowed by phylogenetic footprinting. Hox, Eya, and Pax are known regulatory factors for Six2 expression (Gong et al., 2007). The secreted molecule Wnt9b and its downstream transcription factor TCF/Lef1 is also known to regulate kidney development (Karner et al., 2011). Transcriptional input motif analysis uncovered Hox motifs in CNS2 and CNS5 and Tcf motifs in CNS3 and CNS5 (Fig. 3 A-C). Pax2, the aberrant expression of which is sufficient to induce ectopic nephric structures, was present in CNS5 (Bouchard et al., 2002). Other motifs, such as Sox9, a regulator of nephric duct branching, and early B-cell factor (Erb1), a regulator of podocyte differentiation and glomerular maturation, were found in CNS5 (Fig. 3C)(Reginensi et al., 2011)(Fretz et al., 2014). Motifs for the components of the SWI/SNF complex, Arid3a and Hltf, were found in CNS2 and CNS5.

CNSs were further analyzed using the motif-finding program MEME to identify the consensus motifs in Six2 nephric enhancers, and two consensus motifs were identified (Bailey et al., 2009) (Fig. 3D). Motif 1 is partially overlapped with the putative Hoxa5-binding motif derived from JASPAR in CNS2 and with Tcf3 in CNS3 (Fig. 3). Motif 2 is partially overlapped with Tcf3 and basic helix-loop-helix family, member e40-binding motif in CNE5 (Fig. 3).

The consensus motifs in Six 2 nephric enhancers driven by the 
TABLE 2

PRIMER SEQUENCES FOR CLONING SIX2 CONSERVED NONCODING SEQUENCES (CNSS)

\begin{tabular}{ll} 
Target & Location in genome assembly \\
\hline X. tropicalis Sxi2-CNS1 & xenTro3,GL172661:1,324,307-1,325,472 \\
X. tropicalis Sxi2-CNS2 & xenTro3,GL172661:1,345,329-1,345,807 \\
X. tropicalis Sxi2-CNS3 & xenTro3,GL172661:1,352,848-1,352,986 \\
X. tropicalis Sxi2-CNS4 & xenTro3,GL172661:1,367,624-1,367,928 \\
X. tropicalis Sxi2-CNS5 & xenTro3,GL172661:1,386,790-1,387,226 \\
X. tropicalis Sxi2-CNS6 & xenTro3,GL172661:1,427,350-1,427,819 \\
X. tropicalis Sxi2-CNS7 & xenTro3,GL172661:1,475,679-1,475,909 \\
X. tropicalis Sxi2-CNS8 & xenTro3,GL172661:1,487,514-1,487,667
\end{tabular}

Primer Sequences

tctagaTTGCCAAATCATTCCAAACCGCTAAAAGTC ggatccGGGAAAGTATTATAAACAGAACAGATGGGTGC

tctagaTCTAGA GAGTCTCTATGCCTTCACTACAAGC ggatccCAAACGTACATGTTTTTGCTGAAT

tctagaTTTGTATTCTGCCTCGGTTACGTTCC ggatccCTTCTATTATATACAAAAGGATTAAATACAGATGTTTCCC tctagaGAAGAGGCCCAAACAACCCCTGATTAATTA ggatccAGATGAATATACGAACCTTGAGTTTTAGTGCTAAAAGC tctagaCTGCTTCCTTCTAAATTCGTTCAGCGTG ggatccCCCTGACTGTCACTTTGGAAATAAAACTGAG ggatccGGAGCAATCTGTCAAGCCAGCCCGG ctgcagATCCTGGCTGGGGCCCCCTG tctagaGCAGGTATCACTGGTGAGGGGG ggatcCTTGTTGAATGTTTTACTCCATCTCCCCACAG tctagaGTCAGGGCCAAATGTATCACCGAG ggatccGTTGGTGCCCTGTTAGTGCTCTG

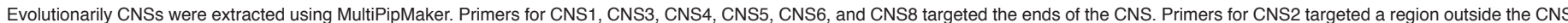

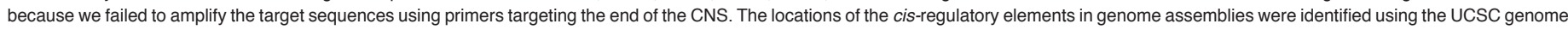
browser. Primer linker sequences are shown in lowercase letters.

MEME motif-finding program illustrated that they are partially overlapped with putative Hoxa5- and Tcf3-binding motifs, whereas the transcription factors that bind to the consensus motifs are unclear. The Hox-Eya-Pax complex and Wnt signaling component Tcf/ Lef1 are known to regulate Six2 expression (Gong et al., 2007) (Park et al., 2012). Therefore, the input by Tcf3, Pax2, and Hoxa5 with unidentified transcription factors that bind to the consensus motifs 1 and 2 may be common features among the Six 2 distal enhances. Although the functional roles of the SWI/SNF complex Arid3a and HItf with regard to Six2 expression are unknown, they are expressed in cells of nephric linage (Petryszak et al., 2014). Hence, Arid3a and HItf are possibly involved in nephric develop- ment. Moreover, although further analysis is required, candidate transcription inputs with motifs that are evolutionarily conserved between humans and frogs may contribute to Six2 expression in both amphibians and mammals during nephrogenesis.

\section{Materials and Methods}

\section{Ethics statement}

All animal care and experiments conformed to the Guidelines of Animal Experiments of Yamagata University, and the experimental protocols were approved by the Animal Research Committee of Yamagata University (26-075).
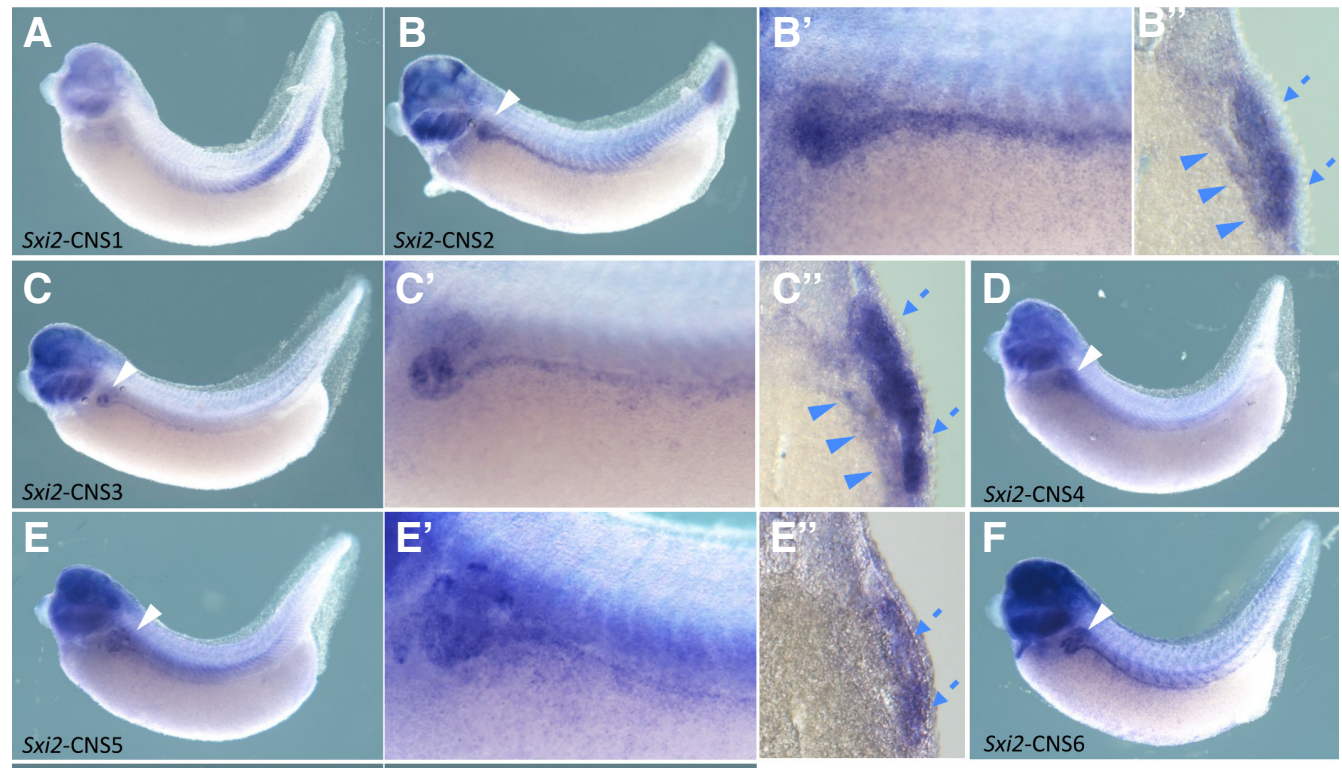

Fig. 2. Green fluorescent protein

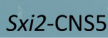

G

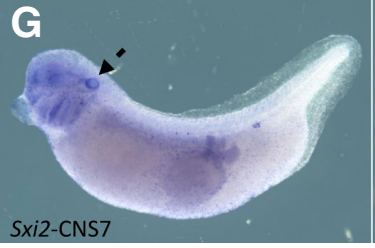

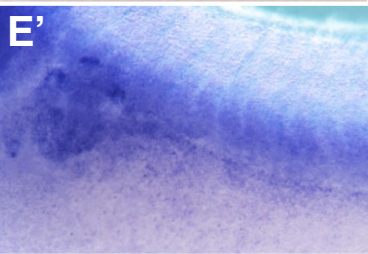

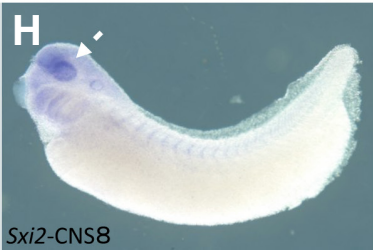

expression pattern driven by Six2-

conserved noncoding sequences. (A-H) Transgenic Xenopus laevis embryos were generated with various reporter constructs, and their expression was analyzed using in situ hybridization. Higher magnification image of pronephric positive embryos ( $\left.\mathbf{B}^{\prime}, \mathbf{C}^{\prime}, \mathbf{E}^{\prime}\right)$. Transverse section of the embryo at the level the pronephros (B",C",E"). White ar rowheads indicate expression in the pronephros. Blue arrows indicate the pronephric tubules and duct, and blue arrowheads indicate the non-glomus mesenchyme. Black and white arrows indicate expression in otic vesicle and the eye tissues, respectively. 
A
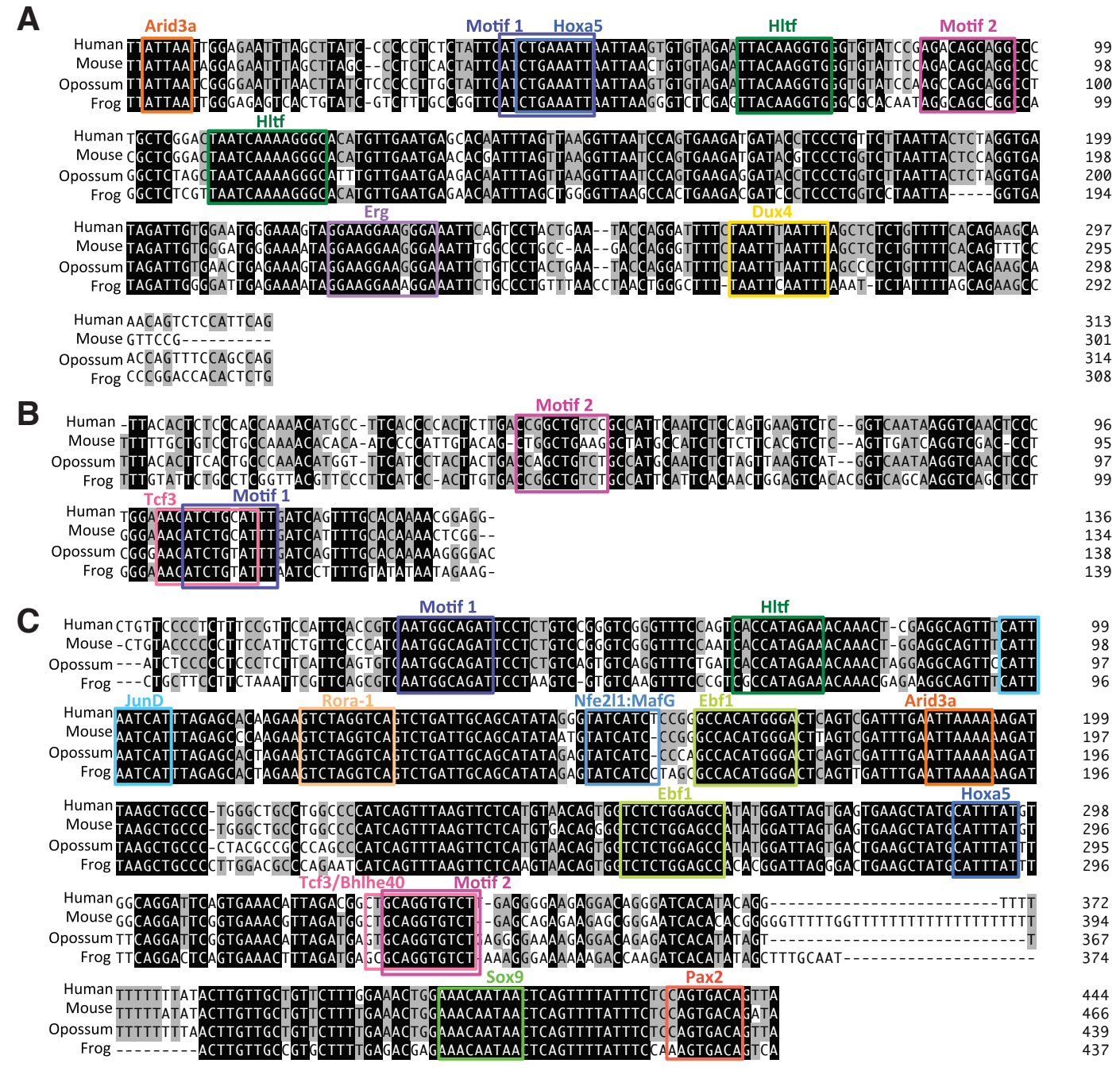

D

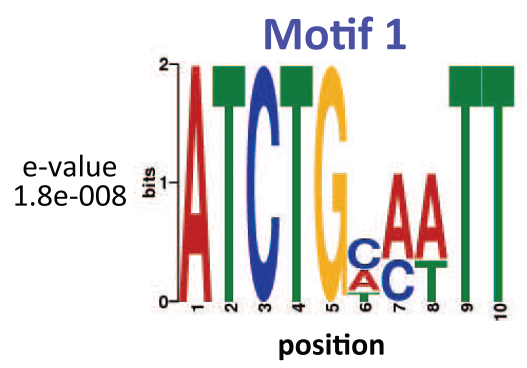

Identification of conserved non-coding sequences

The 364-kb genomic sequence of the human SIX2 locus (hg19chr2_45112852-45477594) and its orthologous sequences in mice (mm10 chr17-84875598-85861124), opossums (monDom5 chr1-652674101-653951640), and $X$. tropicalis (Xenbase 7.1, scaffold_5_108443461-108867602) were downloaded from the UCSC Genome Browser and Xenbase Genome Browser. These sequences were aligned using MultiPipMaker (Schwartz, 2000).

\section{Construction of reporter plasmids}

The green fluorescent protein (GFP) reporter plasmid carrying a chicken $\beta$-actin basal promoter $(-55$ to +53$)$ was previously described as $\beta$-GFP (Ogino and Ochi, 2009). The CNSs were amplified from $X$. tropicalis genomic DNA by PCR and cloned into $\beta$-GFP reporter vectors. The primer

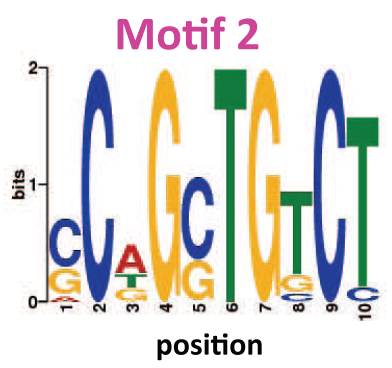

Fig. 3. Phylogenetic footprinting analysis for Six2nephric enhancers. (A-C) Alignment of Six2 with conserved noncoding sequence 2 (CNS2) (A), CNS3 (B), and CNS5 (C). Potential transcription factorbinding motifs in nephric enhancers were extracted using JASPAR ver. 5. Among them, candidates expressed in nephric tissues were selected and further analyzed by phylogenetic footprinting. (D) Sequence logo representation of the consensus motifs in all nephric enhancers. The size of the characters represents the relative frequency of the corresponding position. Sequence of motif 1 in CNS5 (C) and motif 2 in CNS2 (A) indicate reverse complement motifs. The colored boxes denote the evolutionarily conserved putative transcription factor-binding motifs.

sequences used in this study are summarized in Supplementary Table 2.

\section{Cloning of $\mathrm{X}$. tropicalis Six2}

A cDNA fragment of Six2 was amplified from a cDNA pool of $X$. tropicalis tailbud embryos (stage 26) using the following primers: XI_Six2_F: ATGTCGATGCTTCCGACTTTTGGC, XI_Six2_R:TTAAGAGCCAAGGTCTACCAAGTTGGATG. The product was introduced into the $X$ hol and $X b a l$ sites of $\mathrm{pCS} 2$ + MT plasmid, and correct cloning was verified by sequencing analysis. The resulting plasmid was linearized by $\mathrm{Ncol}$ and used as the template for RNA probe synthesis.

\section{Transgenic reporter assay}

Transgenic Xenopus laevis embryos were generated by the sperm nuclear transplantation method with oocyte extracts (Kroll and Amaya, 
1996). The manipulated embryos were cultured until stages $35-36$, and all normally developed embryos were subjected to in situ hybridization to examine their GFP expression with maximum sensitivity. All CNS-carrying reporters were tested at least three times. The frequency of GFP expression varied depending on the constructs, but all constructs exhibited a reproducible expression pattern. The CNSs that drove nephric expression in more than $15 \%$ of the examined embryos were defined as nephric enhancers.

\section{Motif analysis for transcription factor-binding sites}

The open-access database JASPAR ver. 5 was used to search for potential transcription factor-binding sites in nephric enhancers (Mathelier et al., 2014). The candidate transcription factors were narrowed on the basis of their expression using the Expression Atlas (Petryszak et al., 2014). CNSs were aligned using ClustalW, and conserved sequences for the candidate transcription factor-binding sites were further analyzed by phylogenetic footprinting (Blanchette and Tompa, 2002). The MEME suite was used to discover the consensus motifs in Six2 nephric enhancers (Bailey et al., 2009).

\section{Acknowledgments}

This work was supported by Grants-in-Aid for Scientific Research from the Japan Society for the Promotion of Science (JSPS) and the Ministry of Education, Culture, Sports, Science and Technology (MEXT) of Japan (Grant Nos. 25124704, 25125716, 24770214, 25124705, and 23570256), as well as grants from CREST (JST to H. Ogino), the Takeda Science Foundation (H. Ochi) and Senri Foundation (H. Ochi). We thank the National BioResource Project of MEXT at Hiroshima University.

\section{References}

BAILEY TL, BODEN M, BUSKE F a, FRITH M, GRANT CE, CLEMENTI L, REN J, LI WW, NOBLE WS (2009). MEME SUITE: tools for motif discovery and searching. Nucleic Acids Res 37: W202-W208.

BAROLO S (2012). Shadow enhancers: frequently asked questions about distributed cis-regulatory information and enhancer redundancy. Bioessays 34: 135-141.

BLANCHETTE M, TOMPA M (2002). Discovery of regulatory elements by a computational method for phylogenetic footprinting. Genome Res 12: 739-748.

BOUCHARD M, SOUABNI A, MANDLERM, NEUBÜSERA, BUSSLINGERM (2002). Nephric lineage specification by Pax2 and Pax8. Genes Dev 16: 2958-2970.

FRETZJ a, NELSONT, VELAZQUEZH, XIY, MOECKELGW, HOROWITZMC (2014). Early B-cell factor 1 is an essential transcription factor for postnatal glomerular maturation. Kidney Int 85: 1091-1102.

GHANBARI H, SEO HC, FJOSE a, BRÄNDLI a W (2001). Molecular cloning and embryonic expression of Xenopus Six homeobox genes. Mech Dev 101:271-277.

GONG K-Q, YALLOWITZ AR, SUN H, DRESSLER GR, WELLIK DM (2007). A Hox-
Eya-Pax complex regulates early kidney developmental gene expression. $\mathrm{Mol}$ Cell Biol 27: 7661-7668.

KARNER CM, DAS A, MA Z, SELF M, CHEN C, LUM L, OLIVER G, CARROLL TJ (2011). Canonical Wnt9b signaling balances progenitor cell expansion and differentiation during kidney development. Development 138: 1247-1257.

KLEINJAN D a, SEAWRIGHT A, MELLA S, CARR CB, TYAS D a, SIMPSON TI, MASON JO, PRICE DJ, VAN HEYNINGEN V (2006). Long-range downstream enhancers are essential for Pax6 expression. Dev Biol 299: 563-581.

KROLL KL, AMAYA E (1996). Transgenic Xenopus embryos from sperm nuclear transplantations reveal FGF signaling requirements during gastrulation. Development 122: 3173-3183.

MATHELIER A, ZHAO X, ZHANG AW, PARCY F, WORSLEY-HUNT R, ARENILLAS DJ, BUCHMAN S, CHEN C, CHOU A, IENASESCU H, LIM J, SHYR C, TAN G, ZHOU M, LENHARD B, SANDELINA, WASSERMAN WW (2014). JASPAR 2014: an extensively expanded and updated open-access database of transcription factor binding profiles. Nucleic Acids Res 42: D142-D147.

OCHI H, TAMAI T, NAGANO H, KAWAGUCHIA, SUDOU N, OGINO H (2012). Evolution of a tissue-specific silencer underlies divergence in the expression of pax2 and pax8 paralogues. Nat Commun 3: 848 .

OGINO H, OCHI H (2009). Resources and transgenesis techniques for functional genomics in Xenopus. Dev Growth Differ 51: 387-401.

PARKJ, MAW, BRIENLLO, CHUNG E, GUOJ, CHENGJ, VALERIUSMT, MCMAHON JA, WONG WH, MCMAHON AP (2012). Six2 and Wnt Regulate Self-Renewal and Commitment of Nephron Progenitors through Shared Gene Regulatory Networks. Dev Cell 23: 637-651.

PETRYSZAK R, BURDETT T, FIORELLI B, FONSECA N a, GONZALEZ-PORTA M, HASTINGS E, HUBER W, JUPP S, KEAYS M, KRYVYCH N, et al., (2014). Expression Atlas update--a database of gene and transcript expression from microarray- and sequencing-based functional genomics experiments. Nucleic Acids Res 42: D926-D932.

REGINENSI A, CLARKSON M, NEIRIJNCK Y, LU B, OHYAMA T, GROVES AK, SOCK E, WEGNER M, COSTANTINI F, CHABOISSIER M-C, SCHEDLA (2011). SOX 9 controls epithelial branching by activating RET effector genes during kidney development. Hum Mol Genet 20: 1143-1153.

SCHWARTZ S (2000). PipMaker---A Web Server for Aligning Two Genomic DNA Sequences. Genome Res 10: 577-586.

SELF M, LAGUTIN O V, BOWLING B, HENDRIXJ, CAIY, DRESSLER GR, OLIVER G (2006). Six2 is required for suppression of nephrogenesis and progenitor renewal in the developing kidney. EMBO J 25: 5214-5228.

WOOLFE A, GOODSON M, GOODE DK, SNELL P, MCEWEN GK, VAVOURI T, SMITH SF, NORTH P, CALLAWAY H, KELLY K, WALTER K, ABNIZOVA I, GILKS W, EDWARDS YJK, COOKE JE, ELGAR G (2005). Highly conserved non-coding sequences are associated with vertebrate development. PLOS Biol 3: e7.

YALLOWITZ AR, GONG K-Q, SWINEHART IT, NELSON LT, WELLIK DM (2009). Non-homeodomain regions of Hox proteins mediate activation versus repression of Six2 via a single enhancer site in vivo. Dev Biol 335: 156-165. 


\section{Further Related Reading, published previously in the Int. J. Dev. Biol.}

Comparative expression analysis of the H3K27 demethylases, JMJD3 and UTX, with the H3K27 methylase, EZH2, in Xenopus Akane Kawaguchi, Haruki Ochi, Norihiro Sudou and Hajime Ogino

Int. J. Dev. Biol. (2012) 56: 295-300

http://dx.doi.org/10.1387/ijdb.113360ak

\section{Understanding the regulatory genome}

M. Eva Alonso, Bárbara Pernaute, Miguel Crespo, José Luis Gómez-Skarmeta and Miguel Manzanares

Int. J. Dev. Biol. (2009) 53: 1367-1378

http://dx.doi.org/10.1387/ijdb.072428ma

Developmental gene network analysis

Roger Revilla-i-Domingo and Eric H Davidson

Int. J. Dev. Biol. (2003) 47: 695 - 703

http://dx.doi.org/10.1387/ijdb.14756345

Towards a molecular anatomy of the Xenopus pronephric kidney A W Brändli

Int. J. Dev. Biol. (1999) 43: 381 - 395

http://dx.doi.org/10.1387/ijdb.10535314

Tissue and developmental distribution of Six family gene products H Ohto, T Takizawa, T Saito, M Kobayashi, K Ikeda and K Kawakami Int. J. Dev. Biol. (1998) 42: 141 - 148 http://dx.doi.org/10.1387/ijdb.9551859

5 yr ISI Impact Factor $(2013)=2.879$

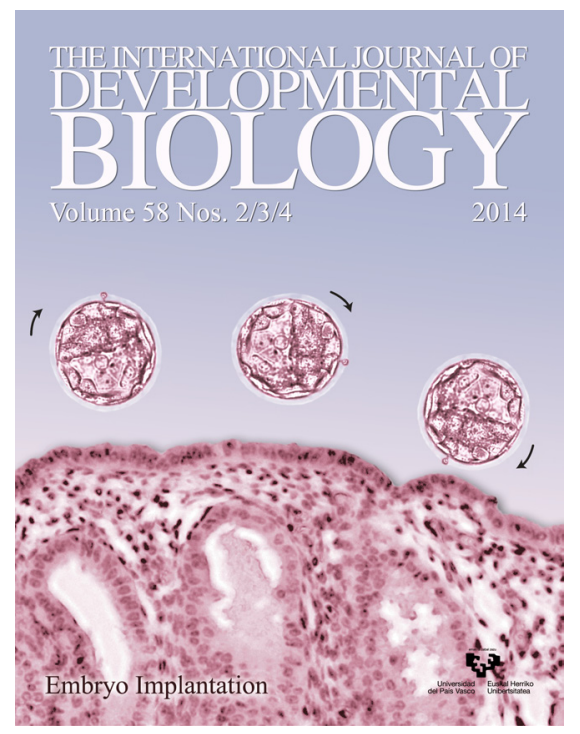

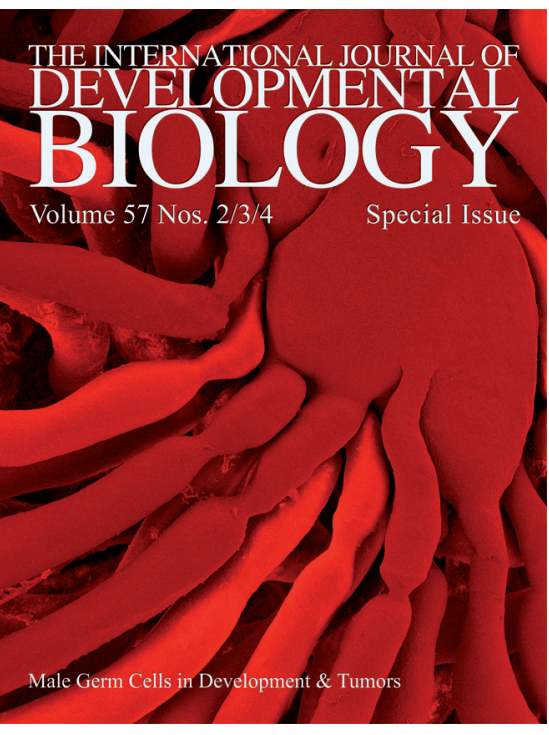
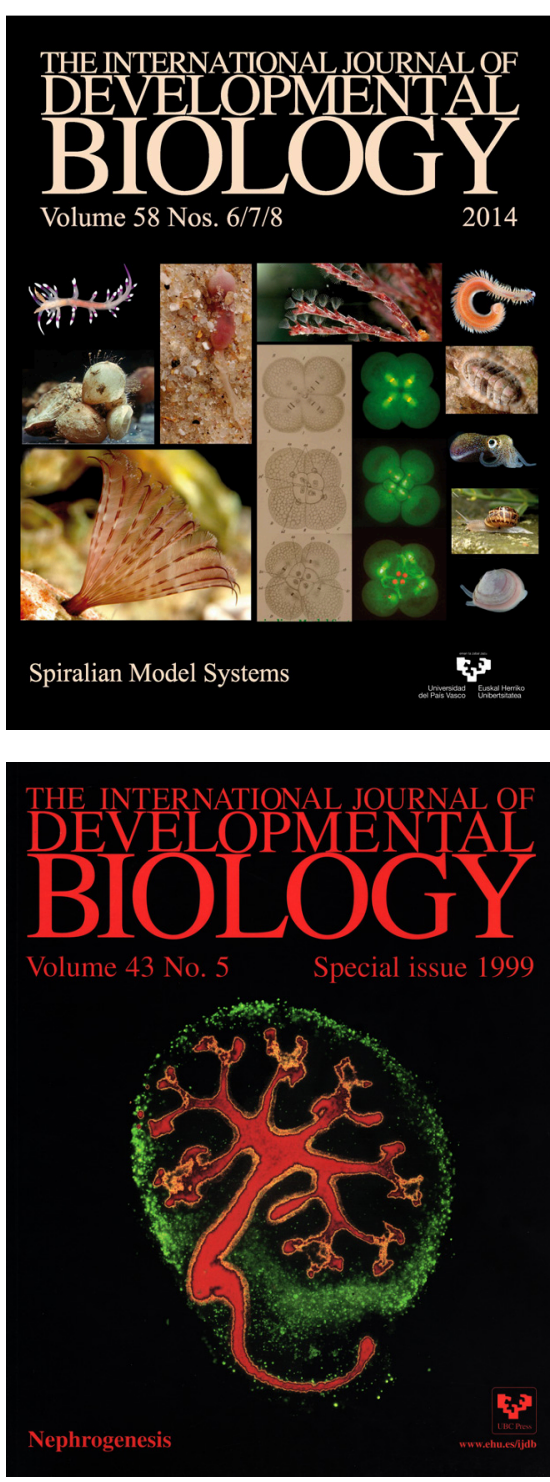